\section{B A Institute of \\ YK Business Administration \\ 六下 \\ Karachi \\ Leadership and Ideas for Tomorrow}

Business Review

Volume 13 Issue 1 January-June 2018

6-15-2018

\title{
Mediating role of psychological ownership on the relationship between psychological capital and burnout amongst university teachers
}

\author{
Adnan Adil \\ Department of Psychology, Ghazali Block, Main Campus, University of Sargodha \\ Anila Kamal \\ National Institute of Psychology, Quaid-i-Azam University, Islamabad \\ Sultan Shujja \\ Department of Psychology, Bhakkar Campus, University of Sargodha \\ Sadia Niazi \\ Department of Psychology, Ghazali Block, Main Campus, University of Sargodha
}

Follow this and additional works at: https://ir.iba.edu.pk/businessreview

\section{(c) (;)}

This work is licensed under a Creative Commons Attribution 4.0 International License.

\section{Recommended Citation}

Adil, A., Kamal, A., Shujja, S., \& Niazi, S. (2018). Mediating role of psychological ownership on the relationship between psychological capital and burnout amongst university teachers. Business Review, 13(1), 69-82. Retrieved from https://doi.org/10.54784/1990-6587.1038

This article is brought to you by iRepository for open access under the Creative Commons Attribution 4.0 License and is available at https://ir.iba.edu.pk/businessreview/vol13/iss1/5. For more information, please contact irepository@iba.edu.pk. 


\title{
Mediating role of psychological ownership on the relationship between psychological capital and burnout amongst university teachers
}

\author{
Adnan Adil • Anila Kamal · Sultan \\ Shujja • Sadia Niazi
}

\begin{abstract}
This research explores the mediating role of psychological ownership on the relationship between psychological capital and burnout. It also examines the differential influences of promotive and preventative psychological ownership on burnout. The purposively selected sample of 500 university teachers, from different public sector universities of Pakistan were subject to the Psychological Ownership Questionnaire, PsyCap Questionnaire and Maslach Burnout Inventory-Educator Survey. The results reveal that psychological capital fosters promotive psychological ownership that reduces the likelihood of burnout. Preventative psychological ownership, however increases the possibility of burnout.
\end{abstract}

Keywords PsyCap · Psychological ownership · Burnout.

\section{Introduction}

The prevalence of high levels of stress and burnout amongst teachers has been acknowledged as a global phenomenon (Jackson et al 2006). According to Bakker et al (2010), recent decades have witnessed many changes in universities, which have deeply influenced the lives of academicians and faculty. Some of these transformations are greater student and staff downsizing, reduction in funding from governments and the use of managerial-style leadership that primarily focuses on efficiency and increased productivity.

Adnan Adil

Department of Psychology, Ghazali Block, Main Campus, University of Sargodha, SargodhaPakistan

E-mail: livespirit786@yahoo.com

Anila Kamal

National Institute of Psychology, Quaid-i-Azam University, Islamabad-Pakistan

Sultan Shujja

Department of Psychology, Bhakkar Campus, University of Sargodha, Sargodha-Pakistan

Sadia Niazi

Department of Psychology, Ghazali Block, Main Campus, University of Sargodha, SargodhaPakistan 
A. Adil et al

The higher student-staff ratio is a corollary to these changes, which has added managerial responsibilities to the job repertoire of university teachers in addition to the increased teaching load and greater pressure to explore financial resources for getting funds for their research projects. This makes rising levels of occupational stress in teachers an inevitable and expected outcome (Biron et al 2008). Furthermore, the gap between supply of competent teachers and the demand from the institutions of higher education is growing. This has modified job perceptions of academics leading to higher levels of stress (Nelson and Simmons 2003).

According to the most established conception, burnout comprises of three components. These are emotional exhaustion, tendency to depersonalize others (perceiving ones self distant from others) and a diminished sense of personal accomplishment (Maslach and Jackson 1982). The key constituent of burnout is emotional exhaustion. This is indicated by reduced energy and a sense that one's affective resources have been depleted. A depersonalized employee is likely to deal with the clients as if they were entities rather than individuals. The attitude of depersonalized employees may become sardonic towards colleagues, clients, and the organization as a whole, leading to an apathetic and indifferent behavior (Cordes and Dougherty 1993). Finally, an employee with a reduced sense of personal accomplishment tends to appraise his/her achievements negatively. This may lead to a deteriorating sense of achievement in relation to his/her job and a reduced sense of job-related capabilities. Most of the time, it is escorted by perceived stagnation or perceived lack of growth in one's career (Cordes and Dougherty 1993).

\section{Psychological capital}

Literature on the buffering influence of a person's strengths and positive capabilities on burnout is quite scarce. Only a few studies have investigated the role of positive psychological resources as protective factors against burnout. The current investigation, therefore, explores one of the most established psychological resources psychological capital or the PsyCap in relation to burnout.

In the context of positive organizational behavior (POB), the constituent elements of psychological capital are measurable, developable, and manageable (Luthans et al 2004). These resources include optimism, hope, resilience, and self-efficacy. To some degree, these resources have already been studied in organizational research in their individual capacity. Initial research on different samples of employees across different cultures has confirmed the unique contribution of PsyCap to the achievement of certain desirable work and organizational outcomes owing to the synergistic effects of its constituents elements, which makes it a source of competitive advantage (Luthans et al 2005).

PsyCap is believed to be instrumental in bridging the gap between a person's actual self and the self that one aspires to be by fostering what one already owns, understands, or who one knows. The preliminary research findings establish the claim that PsyCap has the potential to improve social and human capital through fostering a positive work attitude (Larson and Luthans 2006). 
This cohesive repertoire for conception, assessment, and development of psychological strengths yields numerous sources of synergistic interactions. Since PsyCap has been conceived as a multidimensional latent construct, it can be drawn from both within and across the psychological resource (Law et al 1998). A common thread runs through the super-ordinate construct of PsyCap. It is reflected in the psychological strengths, optimistic appraisal of any given situation, and the chances of gleaning success through one's consistent effort, steady struggle, and determination (Luthans et al 2008).

\subsection{Burnout and PsyCap}

In the profession of teaching, Cooper and Hart (2001) observed that occupational stress was one of the major causes of burnout, which jeopardizes teachers' psychological well-being. Numerous pieces of research evidence suggest that stress is related to various negative psychological states. For instance, within the professional realm of teaching, occupational stress has consistently been found as a predictor of poor well-being, low moods, depression, turnover intentions, and anxiety (Pawan et al 2003). Therefore, occupational stress can be conceived as a major determinant of poor psychological health.

It is noteworthy that findings of pertinent research support the supposition that PsyCap may act as a buffering agent and reduces the negative effects of stress on employees' states of well-being (Luthans et al 2004; Page and Donohue 2004). More specifically, psychological capital might play a mediating role in the context of stressful professions (Luthans et al 2008; Kong 2009). Moreover, PsyCap has been demonstrated as a personal strength, effective in combating occupational stress and its corollaries such as burnout and turnover (Avey et al 2009).

An indigenous study involving employees of the banking and telecommunication sectors identified PsyCap as a negative predictor of self-reported job-related stress and a positive predictor of supervisor-rated innovative job performance (Abbas and Raja 2015). The findings of the aforementioned study elucidate that even in the case of challenging and stressful professions such as university teaching, PsyCap may foster desirable and positive psychological experiences.

Several empirical pieces of evidence support a negative association between burnout and PsyCap. For example, in a sample of nurses, Laschinger and Grau (2012) observed that bullying and emotional exhaustion were inversely related to PsyCap. They also noted that person-job fit mediated the relationships of PsyCap with bullying, mental and physical health, and burnout. The need of fostering PsyCap has been emphasized by Wang et al (2012) because psychological capital may buffer the positive association between burnout and work-family conflict, resulting in happier and more satisfied employees.

\subsection{Psychological ownership}

Avey et al (2009) asserted that psychological ownership has its roots in the evolving theory of positive organizational behavior (POB). Psychological own-

Business Review: (2018) 13(1):69-82 
A. Adil et al

ership is not only measurable, developable, and manageable as per (Luthans 2002a,b) criteria, it also entails a vibe of positivity and a desire for accomplishment in the workplace. Therefore, it can also be conceived as a positive psychological resource. Avey et al (2009) emphasized the need for further refinement in the theory and methodology pertaining to the study of psychological ownership. Thus, being the psychological capacities, psychological ownership, and PsyCap should be positively related.

Pierce et al (2001) suggested that ownership feelings are inborn among humans, which is in line with pertinent literature on the psychology of ownership and possession. The sense of ownership can be developed for both real and immaterial objects and it may have a significant influence on one's affect, attitude, and behavior. These interpretations yield important theoretical points for indepth theoretical understanding of ownership and its probable outcomes.

Van Dyne and Pierce (2004) suggested that the phenomenon of psychological ownership involves the development of possessive feelings towards the target. Psychological ownership may explain a unique variability in work-related outcomes beyond the influence of already recognized attitudinal variables such as commitment and satisfaction. Therefore, possessiveness as the hallmark of psychological ownership not only serves to augment our understanding of employee's attitudes and behaviors on the job but it also differentiates it from other job-related constructs.

In other words, we may conclude that possession, the theoretical ground of psychological ownership is quite unique owing to which it should have the potential of explaining unique variance in work-related outcomes. For Pierce et al (2001), the sense of possession is the key feature of psychological ownership owing to which an object is possessively experienced.

\subsection{Preventative and promotive psychological ownership}

Psychological ownership can be conceived as having two independent forms in terms of Higgins (1998) regulatory focus theory. One may distinguish between promotion focused psychological ownership and prevention-focused psychological ownership. According to Higgins, there are two types of self-regulation systems and individuals regulate themselves through either of them. The one who regulates himself through promotion focused self-regulation system is more ambitious, more daring in terms of risk-taking in his/her endeavors and more achievement-oriented. On the contrary, the one who regulates himself through the preventative system of self-regulation is more anxious and irritated because s/he is more apprehensive of his/her duties and responsibilities (Kark and Van Dijk 2007).

The application of Higgins' theory in terms of psychological ownership suggests that owing to different regulatory foci, individuals are likely to develop a different sense of possession for the target. For instance, a supervisor with promotive psychological ownership is likely to believe that organizational development is personally meaningful to him/her, owing to which s/he shares and imparts his/her skills and knowledge to other colleagues and the subordinates. 
In contrast, according to Avey et al (2009) a supervisor with preventative regulatory focus is more likely to guardedly scrutinize and hide information from others to avoid change and ensure stability.

\subsection{Work outcomes and psychological ownership}

Owing to the novelty of the psychological ownership construct, empirical evidence on its association with important work-related outcomes is yet to be accumulated. It is noteworthy that the available research provides support for the positive influence of psychological ownership on certain desirable work behaviors and attitudes. For example, Md-Sidina, Sambasivana, and Muniandya (2009) observed a positive association of psychological ownership with organizational commitment and satisfaction with the job in a sample of Malaysian university teachers. They also noted the positive effect of psychological ownership on teacher performance in terms of professional services, teaching, and research. In a similar vein, Van Dyne and Pierce (2004) noticed psychological ownership as a positive precursor of an employee's desirable attitude in their three field studies across diverse samples.

However, Mayhew et al (2007) observed that psychological ownership could not predict in-role and extra-role performance although it positively predicted satisfaction with one's job and commitment to one's organization. In an indigenous sample of employees of the telecommunication sector, Ghafoor et al (2011) demonstrated that transformational leadership might enhance followers' sense of psychological ownership, which might lead to improved job performance and work engagement.

The authors of the present research were unable to find any published study on the relationship between burnout and psychological ownership. However, the findings of the previous research (as reviewed above) might be extrapolated to infer that promotive psychological ownership is negatively related to burnout since it has consistently been linked with job satisfaction, performance, commitment, and work engagement. On the other hand, as noted by Kark and Van Dijk (2007), individuals with the preventative mode of ownership are at a higher risk of anxiety and irritation, which may culminate in the form of burnout.

In lieu of the aforementioned review of literature, the following hypotheses were formulated for the present study:

1. PsyCap is negatively associated with burnout.

2. PsyCap is negatively related to preventative ownership and positively associated with promotive ownership.

3. Promotive ownership is negatively and preventative ownership is positively related to burnout.

4. The relationship between burnout and PsyCap is mediated by promotive and preventative ownerships.

Business Review: (2018) 13(1):69-82 
A. Adil et al

\section{Method}

\subsection{Sample}

The sample of the present research included 500 university teachers who were purposively selected from different public sector universities of Islamabad and the Punjab province. The participants' age ranged from 23 to 60 years $(\mathrm{M}=$ $31.76, \mathrm{SD}=7.19)$. The participants had a minimum of 16 years of education. All the participants had at least one year of job experience as a university teacher $(\mathrm{M}=5.67, \mathrm{SD}=6.21)$.

The sample comprised of 197 teachers from the faculty of pure sciences and 303 teachers from the faculty of arts and social sciences. 121 teachers had Ph.D. degrees in their relevant subjects, 121 had MS/MPhil degrees, and the rest had BS or Masters degrees.

In terms of job designations, 54 participants were research associates, 293 were lecturers, 134 were assistant professors, and the rest were associate professors. 274 participants were married and the remaining were single. 289 participants held regular faculty positions while the rest were contractually employed.

\subsection{Measures}

\subsubsection{PsyCap Questionnaire}

The 24-item PsyCap Questionnaire $(\alpha=.95)$ was used to operationalize PsyCap on a 6-point Likert-type scale. Measures of internal consistency for the subscales ranged from .78 to .92 among a sample of employees belonging to diverse professions and organizations (Luthans et al 2007).

For this research, the second order factorial structure of the PsyCap Questionnaire was confirmed through CFA with excellent model fit indices $\chi^{2}(160)$ $=283.39, \mathrm{SRMR}=.033, \mathrm{RMSEA}=.038, \mathrm{NFI}=.94, \mathrm{GFI}=.97, \mathrm{CFI}=.97$. Each of the first order factors (optimism, self-efficacy, hope, and resilience) was constituted by its corresponding six items. All first order and second order factor loadings were $>.50$. A high degree of PsyCap is indicated by high scores on the questionnaire.

\subsubsection{Psychological Ownership Questionnaire (POQ)}

The 16-item Psychological Ownership Questionnaire (POQ) was administered on the participants in order to measure promotive and preventative psychological ownership on a 6-point Likert scale. Item numbers 1-4 assessed preventative while the rest of the items measured promotive psychological ownership. No item of the questionnaire was reversely coded.

A high degree of ownership is reflected by a high score on the POQ. A high score on POQ indicates greater feelings of psychological ownership and vice versa. The reliabilities of both promotive $(\alpha=.91)$ and preventative ownership $(\alpha=.83)$ were quite satisfactory (Avey et al 2009). 
For this research, the second order factorial structure of the promotive psychological ownership subscale of POQ was confirmed through CFA with excellent model fit indices $\chi^{2}(46)=80.49, \mathrm{GFI}=.97, \mathrm{CFI}=.97, \mathrm{NFI}=.96$, RMSEA $=.038, \mathrm{SRMR}=.035$. The first order factors were accountability, self-efficacy, self-identity, and sense of belongingness. All first order and second order factor loadings were $>.50$.

\subsubsection{Maslach Burnout Inventory-ES}

The 22-item Maslach Burnout Inventory-Educator Survey (MBI-ES, = .84; Maslach et al (1996)) was used to operationalize burnout on a 7-point Likert rating scale. The indicators of personal accomplishment subscale were reversed. The average coefficients of internal consistency as reported in the metanalysis of Aguayo, Vargas, Fuente, and Lozano (2011) were .78 for personal accomplishment, .71 for depersonalization, and .88 for emotional exhaustion.

This research confirms the factorial structure of the MBI-ES with excellent model fit indices $\chi^{2}(122)=224.29, \mathrm{GFI}=.95, \mathrm{CFI}=.95, \mathrm{NFI}=.92$, RMSEA $=.037, \mathrm{SRMR}=.047$. All first order and second order factor loadings were $>$ .50 .

\section{Results}

The data analysis for this research has been carried out in two steps. In step I, measurement models were tested for their goodness of fit and in step II, the proposed structural model was tested. Findings of data screening suggested that the data did not have any multivariate outliers.

Table 1 presents the correlation matrix along with descriptive statistics and coefficients of internal consistency, which indicate that all variables were reliably operationalized and were related to one another in the hypothesized directions. None of the values of the coefficients of skewness was aberrantly high, which suggests that the distribution of variables was symmetrical.

Table 1: Correlations, descriptive statistics, and coefficients of internal consistency $(\mathrm{N}=500)$

\begin{tabular}{|c|c|c|c|c|c|c|c|c|}
\hline Variables & M & $\mathrm{SD}$ & $\alpha$ & $S k^{a}$ & 1 & 2 & 3 & 4 \\
\hline PsyCap & 101.47 & 12.26 & 0.91 & -0.49 & & $.60 * * *$ & $-.09 *$ & $-.45 * * *$ \\
\hline Promotive ownership & 57.68 & 8.29 & 0.93 & -0.78 & & & 0.002 & $-.35 * * *$ \\
\hline Preventative ownership & 12.66 & 4.62 & 0.88 & 0.18 & & & & $.14^{* *}$ \\
\hline Burnout & 32.3 & 15.61 & 0.79 & 0.52 & & & & \\
\hline
\end{tabular}

Standard error of skewness $=.11 .{ }^{*} \mathrm{p}<.05 .{ }^{* *} \mathrm{p}<.01, * * * \mathrm{p}<.001$

\subsection{Measurement model}

The proposed measurement model was constituted by four correlated factors including promotive psychological ownership, psychological capital, preventative

Business Review: (2018) 13(1):69-82 
A. Adil et al

psychological ownership, and burnout. This model was compared with threefactor (both types of psychological ownership merged into one factor) and singlefactor models. The findings (see table 2) indicate that in comparison with the two competing models, the proposed measurement model fits significantly better to the data. The poorest fit of the single factor solution i.e., Harman's single factor test (Podsakoff et al., 2003) indicates the lack of mono-method bias.

Table 2: Indices of model fit for the measurement model $(\mathrm{N}=500)$

\begin{tabular}{lcccccccccc}
\hline Models & $\chi^{2}$ & $d f$ & \multicolumn{2}{l}{ Fit Indices } & & & \multicolumn{3}{c}{$\Delta \chi^{2}$} & $\Delta d f$ \\
\hline & & & GFI & AGFI & CFI & NFI & RMSEA & St.RMR & \\
\hline I & 1555 & 134 & 0.74 & 0.64 & 0.65 & 0.64 & 0.14 & 0.16 & - & - \\
II & 606.77 & 131 & 0.88 & 0.82 & 0.87 & 0.85 & 0.08 & 0.12 & $948.25^{*}$ & 3 \\
III & 274.78 & 127 & 0.96 & 0.93 & 0.96 & 0.95 & 0.047 & 0.058 & $331.99^{*}$ & 4 \\
\hline
\end{tabular}

$* \mathrm{p}<.001$

\subsection{Structural model}

The proposed structural model fits well to the data as indicated by various fit indices and the non-significant $\chi^{2}$ value (RMSEA $=.026$, pclose $=.86$, standardized RMR $=.023$; NFI, CFI, and GFI > .95) (see figure 1). Table 3 and

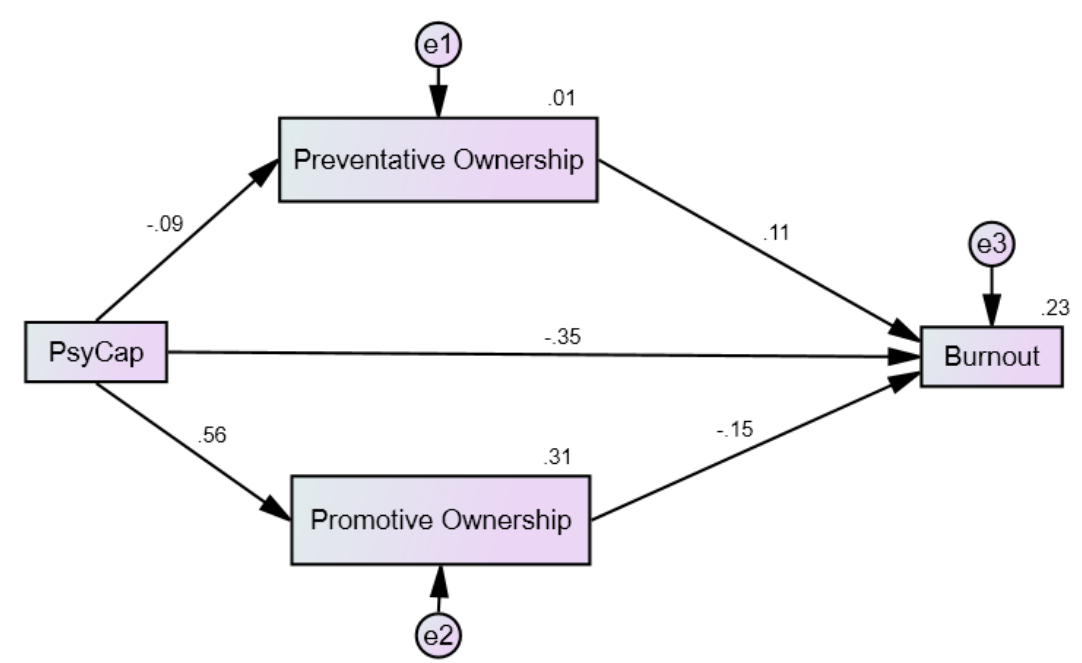

Fig. 1: Schematic presentation of the structural model

All standardized path coefficients were significant $(\mathrm{p}<.05) \chi^{2}(1)=2.07 \mathrm{p}>.05$, GFI=0.99, $\mathrm{CFI}=0.99 \mathrm{NFI}=0.99, \mathrm{RMSEA}=.026$

figure 1 indicate that psychological capital positively predicts promotive ownership and it has direct negative effects on burnout and preventative ownership. 
Promotive ownership demonstrates direct negative effect whereas preventative ownership demonstrates direct positive effect on burnout.

Finally, the indirect effect of PsyCap through preventative ownership as well as promotive ownership on burnout is negative. PsyCap leads to enhanced levels of promotive ownership, which in turn reduces the likelihood of burnout. In contrast, PsyCap reduces the degree of preventative ownership, which in turn results in reduced levels of burnout.

Table 3: Standardized path coefficients for direct and indirect effects $(N=500)$

\begin{tabular}{|c|c|c|c|c|c|}
\hline Predictor Variables & Criterion Variables & $\beta$ & $C I_{95}$ & & $p$ \\
\hline & & & LL & UL & \\
\hline PsyCap & Promotive Ownership & 0.56 & 0.44 & 0.62 & 0.001 \\
\hline PsyCap & Preventative Ownership & -0.09 & -0.17 & -0.02 & 0.006 \\
\hline PsyCap & Burnout & -0.35 & -0.44 & -0.26 & 0.001 \\
\hline Promotive Ownership & Burnout & -0.15 & -0.26 & -0.03 & 0.049 \\
\hline Preventative Ownership & Burnout & 0.11 & 0.04 & 0.16 & 0.001 \\
\hline $\begin{array}{l}\text { PsyCap through Promotive Own- } \\
\text { ership }\end{array}$ & Burnout & -0.01 & -0.03 & -0.003 & 0.006 \\
\hline $\begin{array}{l}\text { PsyCap through Preventative } \\
\text { Ownership }\end{array}$ & Burnout & -0.08 & -0.14 & -0.02 & 0.018 \\
\hline
\end{tabular}

\section{Discussion}

The findings confirm the first hypothesis as PsyCap has demonstrated significant direct negative effect on burnout. This finding is very salient as teaching at the university level is one of those professions that is most vulnerable to burnout. Our findings suggest that burnout among university teachers can be prevented through fostering their PsyCap.

Schaufeli and Bakker (2001) found that some of the employees may not develop burnout despite being exposed to high work demands because they may possess certain psychological strengths and characteristics that act as a shield against burnout. PsyCap is an influential personal strength that helps to stay determined in the face of challenging work environment or hard circumstances.

According to Yardley (2012), PsyCap is negatively associated with burnout among employees. An individual with poor PsyCap lacks the protective factors or the buffering cushions that may protect him/her from the aversive consequences of being stressed in one's occupation.

Yardley (2012) further asserts that in case of a deficient buffering system, a person's capability of reframing negative events into positive situations through the use of psychological resources may dwindle. If s/he invests him/herself physically, emotionally, and mentally in the job and still receives disappointing outcomes, the energy will most likely culminate in burnout. For universities, the evidence that PsyCap has the potential of reducing burnout provides a viable mechanism for the prevention or regulation of burnout.

The second hypothesis is also supported as findings reveal that the direct

Business Review: (2018) 13(1):69-82 
effect of PsyCap on promotive ownership is positive whereas the same for the preventative ownership is negative. It also supports the suggestion of Avey et al (2009) that promotive psychological ownership should be integrated within the research paradigm of $\mathrm{POB}$.

The results of this research illustrate that promotive ownership might be considered as a psychological resource (see Fredrickson, 2003; Hobfoll, 2002). Although promotive ownership has not extensively been explored in relation to POB, several justifications can be put forwarded that indicate its strong link with POB. It can be fostered, assessed, capitalized upon, and regulated for competitive advantage just like other psychological resources.

Results regarding the association between psychological ownership and burnout were supportive of the 3rd and 4th hypotheses. Psychological ownership demonstrated a partial mediation between psychological capital and burnout. We could not identify any published research that has explored the association between burnout and PsyCap. Therefore, the results of this research should be taken as exploratory in nature.

As a component of PsyCap, self-efficacy provides a pertinent line of reasoning as supportive evidence to this inverse association. Self-efficacy is a salient component of promotive ownership and numerous pieces of empirical evidence have established an inverse relationship between burnout and self-efficacy. The reduced personal accomplishment dimension of burnout appears to be the polar opposite of self-efficacy. An employee with a high degree of psychological ownership for his/her organization not only feels confident that s/he has been equipped with the essential skills and capabilities necessary for the attainment of organizational objectives but $\mathrm{s} /$ he may also translate this confidence into work-related behaviors such as in-role and extra-role performance. The need for effectance - a need that spawns psychological ownership, provides impetus for this translation of self-efficacy into superior job-related performance.

As far as the relationship of promotive and preventative ownerships with burnout are concerned, the present research is exploratory in nature. Higgin's theory of regulatory foci provides the theoretical framework for conceptualizing psychological ownership in two distinct forms. For Higgins, self-regulation is the method or the process of goal selection and humans pursue their goals through prevention-focused and promotion-focused regulatory mechanisms.

Kark and Van Dijk (2007) observed that people whose predominant mode of regulation is prevention focused are likely to be very cautious about their assigned roles, responsibilities, and obligations and they remain anxious, irritated, and concerned about ensuring the baseline standards of performance. They are more concerned about rules and regulations and are motivated to avoid punishment or failure. Therefore, meeting deadlines and execution of the assigned task in the prescribed and routine manner without any innovation or novelty becomes important for employees having a high degree of preventative ownership. This makes their work non-challenging, which does not require a high degree of absorption, vigor, and dedication i.e., they perform their work as a routine without experiencing work engagement. Such employees are unlikely to share their knowledge, skills, and wisdom with their colleagues or subordinates because they fear that they can be deprived of their social, intellectual, 
or managerial status in the organization if someone else learns their skills and demonstrates better performance than their own. Under pressing job demands with relatively scarce resources, such employees are an easy prey to burnout.

In contrast, people who predominantly use the promotion-focused regulatory system are daring in taking risks because they are industrious, ambitious, and desirous of going beyond the bare minimum performance standards in order to demonstrate their excellence. A promotion-focused manager or supervisor is likely to share his/her knowledge, skills, and wisdom with his/her colleagues since $\mathrm{s} /$ he believes in team performance and identifies his/her career success with organizational development. For such an employee, development and expansion of the organization is personally gratifying. Therefore, as a personal resource, promotive ownership is likely to lead to an engaged employee, which is a state of personal fulfillment that has been conceived as an antithesis to burnout.

\section{Conclusion and implications}

This research is one of the pioneering researches in Pakistan that has explored the associations between preventative ownership, promotive ownership, PsyCap, and burnout. Owing to its exploratory nature in terms of the variables being studied and the population being chosen, this study has yielded certain imperative implications that can be instrumental in developing a more efficient system of human resource development and management in our indigenous institutions of higher education.

This research has delineated the practical approach of POB in the work milieu of Pakistani universities. Our results demonstrated that PsyCap has the potential of reducing the chance of burnout in university faculty. Therefore, in order to prevent and combat burnout, PsyCap should be fostered among faculty so that we can have engaged, enthusiastic, and dedicated human capital in our universities that can surpass the conventional role of teaching and transpire as true mentors. Therefore, the faculty development programs at our institutions of higher education must evolve some modules that aim at the development of PsyCap in our teachers.

Findings of this study in relation to psychological ownership also offer very important implications for the higher education sector of Pakistan. The mediating role of promotive ownership between PsyCap and burnout indicates that a work environment conducive to the development of promotive ownership is one of the routes through which PsyCap may prevent burnout among university teachers. A work milieu replete in procedural justice and sufficient job/organizational resources is likely to hatch a sense of promotive ownership while an organizational climate characterized by the competitiveness may develop feelings of territoriality or preventative ownership.

Business Review: (2018) 13(1):69-82 
A. Adil et al

\subsection{Limitations of the study}

Before interpreting the findings of this study, certain limitations should be considered. The present study was conducted on full-time university faculty. Although this research has scrutinized certain constructs of POB and their influence on job-related outcomes such as burnout, yet it is noteworthy that the job dynamics of university faculty are quite different from those of the corporate or business sector. Therefore, one should be cautious while generalizing the findings of the present study beyond the population of university teachers of Pakistan.

The participants of the current research were purposively recruited from public sector universities of the capital territory and the province of Punjab. Institutions of higher education in other provinces of the country might have a different organizational climate owing to diverse provincial cultures within Pakistan. Furthermore, public sector universities are distinguishable from those of the private sector on a number of dimensions such fee structure, infrastructure, qualification of the faculty, quality of education, and so on. Therefore, the sample of this study cannot be conceived as a national representative sample of university teachers.

Lecturers and assistant professors were over represented while the senior faculty positions were under represented in the sample of this study. Results might have been different if the data had been collected from senior faculty.

Owing to the use of self-report measures, this study may inherit the peril of mono-method bias and inflated relationships among the variables. However, in pertinent literature, self-report measures constitute the most prevalent way of the operationalization of the constructs of the study. Moreover, none of the correlations in the correlation matrix was too high to signal the inflated rate of relationship. The Herman's single factor test of the measurement model of this research also evidenced that common method variance is not a salient issue for this investigation.

Lastly the cross-sectional design of the research precludes causal interpretation of the findings. Reciprocal relationships are quite possible among various constructs of the present study. However, the choice of the predictor and outcome variables was in strict compliance with relevant literature.

\subsection{Suggestions for future research}

A larger, more diverse, and more representative sample is needed to augment the external validity of the findings of the current research. Therefore, future research should recruit a representative national sample of faculty from both private and public universities across the country. Moreover, faculty members of all designations should have a proportional representation in the sample.

A more occupationally diverse sample should be recruited for further studies so that the results of the current study can be endorsed across various professional categories. This may also help in discerning how job-specific demands may impact the associations among various constructs of POB. 
Longitudinal design in future studies may help in identification of a causal pattern of association among various variables. Aslo different constructs of POB can be explored at their fact level in future studies. It would be interesting to note whether any components of the superordinate constructs such as PsyCap or promotive ownership are interrelated in a different fashion than their corresponding core construct. This would be instrumental in explaining the fine dynamics of associations among different variables.

Each organization is characterized by its own unique organizational culture. The potential influence of organizational culture on work-related constructs is undeniable. The current research did not control the influence of organizational culture. Future studies can explore how organizational culture might foster or curb the development of psychological ownership and PsyCap.

\section{References}

Abbas M, Raja U (2015) Impact of psychological capital on innovative performance and job stress. Canadian Journal of Administrative Sciences/Revue Canadienne des Sciences de l'Administration 32(2):128-138

Avey JB, Avolio BJ, Crossley CD, Luthans F (2009) Psychological ownership: Theoretical extensions, measurement and relation to work outcomes. Journal of Organizational Behavior: The International Journal of Industrial, Occupational and Organizational Psychology and Behavior 30(2):173-191

Bakker AB, Boyd CM, Dollard M, Gillespie N, Winefield AH, Stough C (2010) The role of personality in the job demands-resources model: A study of australian academic staff. Career Development International 15(7):622-636

Biron C, Brun JP, Ivers H (2008) Extent and sources of occupational stress in university staff. Work 30(4):511-522

Cooper C, Hart P (2001) Occupational stress: Towards a more integrated framework. Handbook of industrial and organizational Psychology Sage

Cordes CL, Dougherty TW (1993) A review and an integration of research on job burnout. Academy of management review 18(4):621-656

Ghafoor A, Qureshi TM, Khan MA, Hijazi ST (2011) Transformational leadership, employee engagement and performance: Mediating effect of psychological ownership. African journal of business management 5(17):7391-7403

Higgins ET (1998) Promotion and prevention: Regulatory focus as a motivational principle. In: Advances in experimental social psychology, vol 30, Elsevier, pp 1-46

Jackson LT, Rothmann S, Van de Vijver FJ (2006) A model of work-related well-being for educators in south africa. Stress and Health: Journal of the International Society for the Investigation of Stress 22(4):263-274

Kark R, Van Dijk D (2007) Motivation to lead, motivation to follow: The role of the selfregulatory focus in leadership processes. Academy of Management Review 32(2):500-528

Kong Y (2009) A study on the relationships between job engagement of middle school teachers and its relative variables. Asian Social Science 5(1):105

Larson M, Luthans F (2006) Potential added value of psychological capital in predicting work attitudes. Journal of leadership \& organizational studies 13(1):45-62

Laschinger HKS, Grau AL (2012) The influence of personal dispositional factors and organizational resources on workplace violence, burnout, and health outcomes in new graduate nurses: A cross-sectional study. International journal of nursing studies 49(3):282-291

Law KS, Wong CS, Mobley WM (1998) Toward a taxonomy of multidimensional constructs. Academy of management review 23(4):741-755

Luthans F (2002a) The need for and meaning of positive organizational behavior. Journal of organizational behavior pp 695-706

Luthans F (2002b) Positive organizational behavior: Developing and managing psychological strengths. Academy of Management Perspectives 16(1):57-72

Business Review: (2018) 13(1):69-82 
A. Adil et al

Luthans F, Luthans KW, Luthans BC (2004) Positive psychological capital: Beyond human and social capital

Luthans F, Avolio BJ, Walumbwa FO, Li W (2005) The psychological capital of chinese workers: Exploring the relationship with performance. Management and Organization Review 1(2):249-271

Luthans F, Youssef CM, Avolio BJ, et al (2007) Psychological capital: Developing the human competitive edge, vol 198. Oxford University Press Oxford

Luthans F, Norman SM, Avolio BJ, Avey JB (2008) The mediating role of psychological capital in the supportive organizational climateemployee performance relationship. Journal of Organizational Behavior: The International Journal of Industrial, Occupational and Organizational Psychology and Behavior 29(2):219-238

Maslach C, Jackson S (1982) Burnout in health professions: a social psychological analysis. en gs. sanders y $\mathrm{j}$ suls. social psychology of health and illness

Maslach C, Jackson S, Leiter M (1996) Burnout inventory manual. Palo Alto, CA: Consulting Psychologists

Mayhew MG, Ashkanasy NM, Bramble T, Gardner J (2007) A study of the antecedents and consequences of psychological ownership in organizational settings. The Journal of social psychology 147(5):477-500

Nelson DL, Simmons BL (2003) Health psychology and work stress: A more positive approach. Handbook of occupational health psychology 2:97-119

Page LF, Donohue R (2004) Positive psychological capital: A preliminary exploration of the construct. Department of management pp 1-10

Pawan F, Paulus TM, Yalcin S, Chang CF (2003) Online learning: Patterns of engagement and interaction among in-service teachers

Pierce JL, Kostova T, Dirks KT (2001) Toward a theory of psychological ownership in organizations. Academy of management review 26(2):298-310

Schaufeli W, Bakker A (2001) Work and well-being: towards a positive approach in occupational health psychology. Gedrag \& Organisatie 14:229-253

Van Dyne L, Pierce JL (2004) Psychological ownership and feelings of possession: Three field studies predicting employee attitudes and organizational citizenship behavior. Journal of Organizational Behavior: The International Journal of Industrial, Occupational and Organizational Psychology and Behavior 25(4):439-459

Wang Y, Liu L, Wang J, Wang L (2012) Work-family conflict and burnout among chinese doctors: the mediating role of psychological capital. Journal of occupational health 54(3):232240

Yardley K (2012) Psychological capital as a positive resource to assist with the organisational outcomes of work family conflict: a thesis presented in partial fulfilment of the requirements for the degree of master of arts in psychology, massey university, albany, new zealand. PhD thesis, Massey University 